\title{
Cavity ring-down spectroscopy measurements of sub-Doppler hyperfine structure
}

\author{
D. A. Long, ${ }^{1}$ D. K. Havey, ${ }^{2}$ M. Okumura, ${ }^{1}$ C. E. Miller, ${ }^{3}$ and J. T. Hodges ${ }^{2}$ \\ ${ }^{1}$ Division of Chemistry and Chemical Engineering, California Institute of Technology, Pasadena, California 91125, USA \\ ${ }^{2}$ Process Measurements Division, National Institute of Standards and Technology, 100 Bureau Drive, Gaithersburg, Maryland 20899, USA \\ ${ }^{3}$ Jet Propulsion Laboratory, California Institute of Technology, 4800 Oak Grove Drive, Pasadena, California 91109, USA
}

(Received 23 March 2010; published 16 June 2010)

\begin{abstract}
Frequency-stabilized cavity ring-down spectroscopy (FS-CRDS) was used to measure magnetic dipole transitions in the $b^{1} \Sigma_{g}^{+} \leftarrow X^{3} \Sigma_{g}^{-}(0,0)$ band of $\mathrm{O}_{2}$. The ${ }^{17} \mathrm{O}$-containing isotopologues show unresolved hyperfine structure due to magnetic hyperfine splitting in the ground state. The sensitivity and stability of FS-CRDS allow for quantitative sub-Doppler measurements of the hyperfine constants, even when the hyperfine splittings are much smaller than the Doppler width. Unlike saturation spectroscopy, this linear absorption technique can be applied to weak transitions and employed to quantitatively measure intensities and line shapes. This method may be an attractive approach for measuring unresolved hyperfine structure in excited electronic states.
\end{abstract}

DOI: 10.1103/PhysRevA.81.064502

PACS number(s): 33.15.Pw, 33.70.Jg

Recent advances in near-infrared spectroscopy have enabled measurements with frequency precision previously only possible in the microwave regime [1-3]. Unfortunately, large near-infrared Doppler widths often obscure any hyperfine structure. While saturation spectroscopy can resolve individual hyperfine components $[2,4]$, it is by its very nature a nonlinear technique. As a result, saturation spectroscopy leads to distortion of the spectral line shape and cannot be employed to quantitatively measure line-shape parameters [5-7]. In addition, saturation spectroscopy is less general than traditional absorption spectroscopy and cannot easily be applied to the study of weak transitions, such as those described herein.

Here we describe an alternate approach to the measurement of hyperfine structure in the near-infrared region: the use of a linear and highly sensitive cavity ring-down spectrometer. In particular, the hyperfine splitting of the ${ }^{17} \mathrm{O}$-containing isotopologues of $\mathrm{O}_{2}$ were measured for magnetic dipole transitions in the $b^{1} \Sigma_{g}^{+} \leftarrow X^{3} \Sigma_{g}^{-}(0,0)$ band. While individual hyperfine transitions are unresolved, the spectra exhibit a sufficiently large signal-to-noise ratio to enable quantitative measurements of hyperfine coupling constants. We note that despite the large number of recent studies which have measured spectroscopic parameters of the $b^{1} \Sigma_{g}^{+} \leftarrow X^{3} \Sigma_{g}^{-}(0,0)$ band for ${ }^{16} \mathrm{O}_{2}$ and the rare isotopologues [9], observation of hyperfine splitting has not previously been reported.

Measurements were made using the frequency-stabilized cavity ring-down spectrometer (FS-CRDS) located at the National Institute of Standards and Technology (NIST) in Gaithersburg, MD. The FS-CRDS system has been described in detail previously [10,11], and therefore only the most important details are given. FS-CRDS differs from other single-mode cw-CRDS spectroscopies by actively stabilizing the intracavity length to an external frequency reference. This length stabilization in turn stabilizes the cavity's free spectral range (FSR). The probe laser frequency is then stepped between successive $\mathrm{TEM}_{00}$ cavity modes, resulting in an accurate, stable, and linear frequency axis. FS-CRDS has been utilized to measure transition frequencies to better than $0.5 \mathrm{MHz}$ [1] and line intensities to better than $0.3 \%$ [12]. Importantly, due to active frequency stabilization, FS-CRDS does not exhibit instrumental broadening and has therefore been able to record $\mathrm{O}_{2}$ Doppler (Gaussian) widths to $143 \mathrm{kHz}$, which is 1 part in 6,000 of the room-temperature Doppler width $(\sim 850 \mathrm{MHz})$ for this band [13].

For the described measurements, the probe was an externalcavity diode laser with a tuning range of 759-771 nm, an output power of $6-10 \mathrm{~mW}$, and a line width of $<1 \mathrm{MHz}(<3 \times$ $\left.10^{-5} \mathrm{~cm}^{-1}\right)$. The frequency reference was a frequencystabilized HeNe laser with a frequency uncertainty of less than $1 \mathrm{MHz}$ over $8 \mathrm{~h}$. Mirrors with $99.98 \%$ reflectivity were used, corresponding to a finesse of $\sim 15,000$. The resulting short-term noise equivalent absorption coefficient was $6 \times$ $10^{-10} \mathrm{~cm}^{-1} \mathrm{~Hz}^{-1 / 2}$, and the cavity FSR was determined to be 201.970(10) MHz. A continuous spectrum was measured over the $R$ branch of the $b^{1} \Sigma_{g}^{+} \leftarrow X^{3} \Sigma_{g}^{-}(0,0)$ band (13126$\left.13165 \mathrm{~cm}^{-1}\right)$ with a step size of $100 \mathrm{MHz}\left(\sim 0.003 \mathrm{~cm}^{-1}\right)$. Sub-FSR frequency steps were enabled by frequency shifting the HeNe reference laser through the use of an acousto-optic modulator in a double-pass configuration [10].

A ${ }^{17} \mathrm{O}$-enriched $\mathrm{O}_{2}$ sample containing $15.23 \%{ }^{16} \mathrm{O}_{2}$, $38.16 \%{ }^{16} \mathrm{O}^{17} \mathrm{O}, 8.28 \%{ }^{16} \mathrm{O}^{18} \mathrm{O}, 25.93 \%{ }^{17} \mathrm{O}_{2}, 11.15 \%{ }^{17} \mathrm{O}^{18} \mathrm{O}$, and $1.25 \%{ }^{18} \mathrm{O}_{2}$ was used. Measurements were performed at a pressure of $236 \mathrm{~Pa}$ (1.77 Torr) and a temperature of $299.5 \mathrm{~K}$. The combined uncertainty (random and systematic) of this temperature measurement is $\sim 28 \mathrm{mK}$ [14]. Spectra were fit using Voigt line-shape profiles, which account for pressure (Lorentzian) and Doppler (Gaussian) broadening. The importance of using the Galatry [15] line profile for high-resolution measurements at higher pressures is well established $[16,17]$; however, due to the low pressures of this study, Dicke narrowing [18] is less than $1 \mathrm{MHz}$ and was therefore neglected. During the fit, the transition position and intensity were floated while the pressure broadening coefficient was constrained to the ${ }^{16} \mathrm{O}_{2}$ known values [12]. We have recently shown that there is no difference between the pressure broadening parameters for the $\mathrm{O}_{2}$ isotopologues to within the experimental uncertainty of $2 \%$ [8]; therefore, at the low pressures utilized herein, the use of the ${ }^{16} \mathrm{O}_{2}$ coefficients for all isotopologues should introduce negligible error. Further experimental details will be given in a separate publication [19].

The $b^{1} \Sigma_{g}^{+} \leftarrow X^{3} \Sigma_{g}^{-}(0,0)$ band is triply forbidden by quantum mechanical electric dipole selection rules but occurs as a magnetic dipole transition [20]. Due to this magnetic dipole character and the necessary change in multiplicity, 
the $b^{1} \Sigma_{g}^{+} \leftarrow X^{3} \Sigma_{g}^{-}(0,0)$ band of ${ }^{16} \mathrm{O}_{2}$ is very weak with a band intensity of only $2.231(7) \times 10^{-22} \mathrm{~cm} \mathrm{molec}^{-1}$ at $296 \mathrm{~K}$ [12]. The rotational levels of $\mathrm{O}_{2}$ are described by three quantum numbers: $N$ is the rotational angular momentum, $S$ is the spin, and $J$ is their sum (i.e., $J=N+S$ ). The $\mathrm{O}_{2}$ ground state is a good Hund's case (b) and is split into three levels $\left(J^{\prime \prime}=N^{\prime \prime}-1, N^{\prime \prime}, N^{\prime \prime}+1\right)$, while the upper state has only $J^{\prime}=N^{\prime}$. For ${ }^{16} \mathrm{O}_{2}$ and ${ }^{18} \mathrm{O}_{2}$, only odd levels of $N^{\prime \prime}$ are allowed due to spin statistics, while all values of $N^{\prime \prime}$ are allowed for the remaining isotopologues. We denote the $b^{1} \Sigma_{g}^{+} \leftarrow X^{3} \Sigma_{g}^{-}(0,0)$ transitions by $\Delta N N^{\prime \prime}, \Delta J J^{\prime \prime}$ with the band divided into four branches ( $P P, P Q, R R$, and $R Q$ ).

While ${ }^{16} \mathrm{O}$ and ${ }^{18} \mathrm{O}$ have a nuclear spin of $I=0,{ }^{17} \mathrm{O}$ has $I=5 / 2$, and therefore the ${ }^{17} \mathrm{O}$-containing isotopologues exhibit hyperfine structure. In the ${ }^{3} \Sigma_{g}^{-}$ground state, this hyperfine splitting is almost entirely magnetic in nature due to the unpaired spins. Miller and Townes demonstrated that any higher order magnetic and nuclear electric quadrupole hyperfine couplings are negligible (i.e., $<1 \mathrm{MHz}$ ) [21]. This ground-state hyperfine structure has been observed in microwave, millimeter, submillimeter, and electron paramagnetic resonance spectroscopy measurements of the ${ }^{16} \mathrm{O}^{17} \mathrm{O}[21-23]$, ${ }^{17} \mathrm{O}_{2}$ [24], and ${ }^{17} \mathrm{O}^{18} \mathrm{O}$ [23] isotopologues.

The upper state $\left({ }^{1} \Sigma_{g}^{+}\right)$is a singlet state and therefore does not exhibit a permanent magnetic dipole. Thus, any magnetic hyperfine coupling is the result of a perturbation and will be extremely weak [25]. Additionally, it can be shown that the electronic structure of this state leads to a weak electric quadrupole coupling (on the order of a few megahertz) [25-27]. Thus, we assume that all observed hyperfine structure is the result of hyperfine splitting within the ${ }^{3} \Sigma_{g}^{-}$ground state. This assumption will introduce at most an error of a few megahertz to the measured hyperfine coupling constants.

For ${ }^{16} \mathrm{O}^{17} \mathrm{O}$ and ${ }^{17} \mathrm{O}^{18} \mathrm{O}$, the hyperfine energies were calculated by Miller and Townes [25] based upon the formalism of Frosch and Foley [28]:

$$
\begin{gathered}
E_{J=N+1}=\frac{\mathbf{I} \cdot \mathbf{J}}{N+1}\left(b+\frac{c}{2 N+3}\right), \\
E_{J=N}=\frac{\mathbf{I} \cdot \mathbf{J}}{N(N+1)}(b+c), \\
E_{J=N-1}=\frac{\mathbf{I} \cdot \mathbf{J}}{N}\left(-b+\frac{c}{2 N-1}\right),
\end{gathered}
$$

where

$$
\mathbf{I} \cdot \mathbf{J}=\frac{F(F+1)-I(I+1)-J(J+1)}{2}
$$

and

$$
\mathbf{F}=\mathbf{I}+\mathbf{J}
$$

The experimentally determined values of the hyperfine coupling constants are $b=-101.441(5) \mathrm{MHz}, c=139.73(3) \mathrm{MHz}$ for ${ }^{16} \mathrm{O}^{17} \mathrm{O}$ and $b=-101.46(1) \mathrm{MHz}, c=139.68(6) \mathrm{MHz}$ for ${ }^{17} \mathrm{O}^{18} \mathrm{O}$ [23]. For ${ }^{16} \mathrm{O}^{17} \mathrm{O}$, the hyperfine coupling constants have been theoretically confirmed using both a simple Slatertype orbital formalism [29] and the multiconfiguration selfconsistent field method [30].
Our spectra do not show resolved hyperfine transitions; however, we do observe as much as $200 \mathrm{MHz}(\sim 1 / 4$ the Doppler width) of line broadening in excess of the Doppler width. We ascribe this broadening to the hyperfine structure. We have previously measured the $\mathrm{O}_{2}$ Doppler width to within $143 \mathrm{kHz}$ [13]; as a result, the observed broadening cannot be attributed to instrumental broadening.

The $R$ branch of the $b^{1} \Sigma_{g}^{+} \leftarrow X^{3} \Sigma_{g}^{-}(0,0)$ band consists of $R R$ and $R Q$ transitions. The $R R$-branch transitions begin in ground-state levels which have $J^{\prime \prime}=N^{\prime \prime}$; thus, their hyperfine splitting is described by Eq. (2). Note that $b$ and $c$ have opposite signs and $(b+c) \sim 38 \mathrm{MHz}$. As a result, minimal hyperfine splitting would be expected for these transitions, especially for large $N^{\prime \prime}$. As can be seen in Fig. 1(a), the $R R$ transitions for each isotopologue exhibit minimal
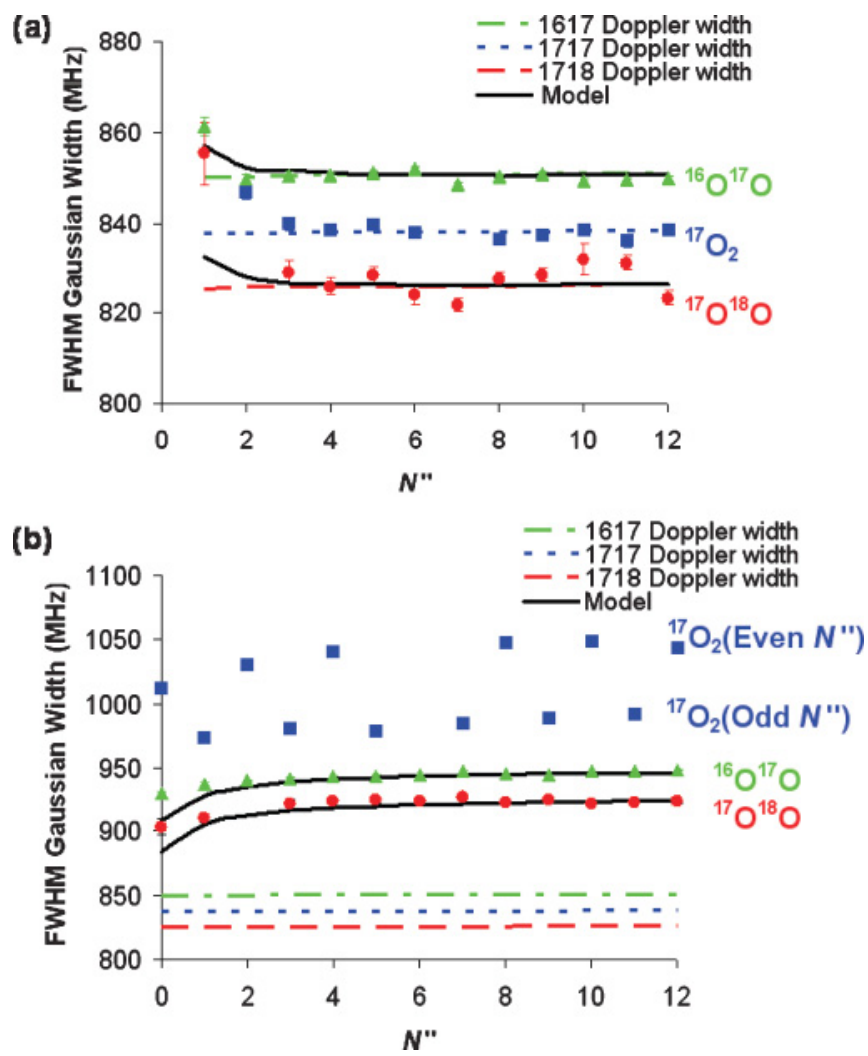

FIG. 1. (Color online) Experimental Gaussian full width at halfmaximum (FWHM) and theoretical Doppler widths (dashed horizontal lines) in megahertz for the ${ }^{17} \mathrm{O}$-containing isotopologues with fit uncertainties. Each experimental Gaussian width was determined by a Levenberg-Marquardt least-squares fit using a single Voigt line shape with the pressure-broadening parameter constrained to the previously determined ${ }^{16} \mathrm{O}_{2}$ value [12]. Note that collisional narrowing is negligible (i.e., $<1 \mathrm{MHz}$ ) at the pressure utilized in this study, although it can be very important at higher pressures [16,17]. Also shown are calculated (solid black lines) broadenings for ${ }^{16} \mathrm{O}^{17} \mathrm{O}$ and ${ }^{17} \mathrm{O}^{18} \mathrm{O}$ based upon the model described in the text. (a) $R R$-branch transitions which exhibit minimal broadening (except for verylow- $N^{\prime \prime}$ transitions). (b) $R Q$-branch transitions exhibit significant broadening due to the large hyperfine splitting (note the different $y$-axis scales between the two figures). The hyperfine splitting of the ${ }^{17} \mathrm{O}_{2}$ transitions exhibit a dependence on the parity of $N^{\prime \prime}$ due to symmetry constraints. For both branches, the model performs well for transitions with $N^{\prime \prime}>1$. 
broadening, with observed Gaussian widths converging to the Doppler width for $N^{\prime \prime}>2$.

The $R Q$ branch begins in ground-state levels having $J^{\prime \prime}=N^{\prime \prime}+1$. In this case, $b$ dominates the observed hyperfine splitting for $N^{\prime \prime}>4$. As a result, hyperfine splittings of hundreds of megahertz are expected, with splittings increasing to an asymptotic limit with increasing $N^{\prime \prime}$. In Fig. 1(b), it can readily be seen that the expected behavior is observed for each of the isotopologues.

For ${ }^{17} \mathrm{O}_{2}$, the parity of $N^{\prime \prime}$ has a large impact upon the observed splitting. ${ }^{17} \mathrm{O}_{2}$ has two $I=5 / 2$ nuclei and therefore has a total nuclear spin of $I_{\text {total }}=5,4,3,2,1,0$. The symmetry of ${ }^{17} \mathrm{O}_{2}$ dictates that odd values of $N^{\prime \prime}$ can only have even values of $I_{\text {total }}$ and even values of $N^{\prime \prime}$ can only have odd values of $I_{\text {total }}$ [24]. Thus, even values of $N^{\prime \prime}$ have $I_{\text {total }}=5,3,1$, and odd values of $N^{\prime \prime}$ have $I_{\text {total }}=4,2,0$. Since larger values of $I_{\text {total }}$ give rise to larger splitting, we would expect even values of $N^{\prime \prime}$ to have a larger splitting [as can be seen in Fig. 1(b)].

The observed broadening can be analyzed with a simple intensity model to determine $b$ and $c$. In the high- $J$ case, only hyperfine transitions with $\Delta J=\Delta F$ are significant, and the intensities of these transitions are roughly proportional to $(2 F+1)$ [25]. This high- $J$ limit was utilized to calculate the relative intensities of the hyperfine structure components for each of the measured transitions. The hyperfine structure positions were calculated as shown previously. The hyperfine spectrum could then be readily calculated.

It can readily be shown algebraically that for all $J=N \pm$ 1,0 ,

$$
\sum_{F}(2 F+1) E_{J}(F)=0,
$$

where each sum is over all allowed $F$ quantum numbers (i.e., $F=I+J, I+J-1, \ldots,|I-J|)$ and $E_{J}$ is defined as in Eqs. (1)-(3). Thus, the calculated hyperfine structure does not shift the spectral center of mass.

For ${ }^{16} \mathrm{O}^{17} \mathrm{O}$ and ${ }^{17} \mathrm{O}^{18} \mathrm{O}$, these calculated frequency splittings and intensities were then utilized to simulate the broadened line shape as a sum of six separate hyperfine profiles whose Gaussian widths were fixed to the theoretical Doppler width for the given isotopologue. As can be seen in Fig. 1, this model reproduces the observed broadening very well for $N^{\prime \prime}>3$. For $N^{\prime \prime} \leqslant 3$, the assumptions made regarding the relative intensities of the hyperfine structure break down and more complicated expressions are required.

Figure 2 shows a measured spectrum which has been fit by a set of six Voigt line profiles (one for each hyperfine component). Each of these hyperfine profiles had its Gaussian width set equal to the theoretical Doppler width and the pressure-broadening parameter fixed to the value determined for ${ }^{16} \mathrm{O}_{2}$ [12]. Thus, the only two fitted parameters were the position of the spectral center of mass and the total integrated area (i.e., all relative hyperfine coupling constants were held fixed). As can be seen in Fig. 2, this model captures the observed behavior to within the baseline noise level.

We were able to determine the $b$ and $c$ hyperfine coupling constants by least-squares fits of the model to our measured spectra. For a fixed value of $N^{\prime \prime}$, it is not possible to determine

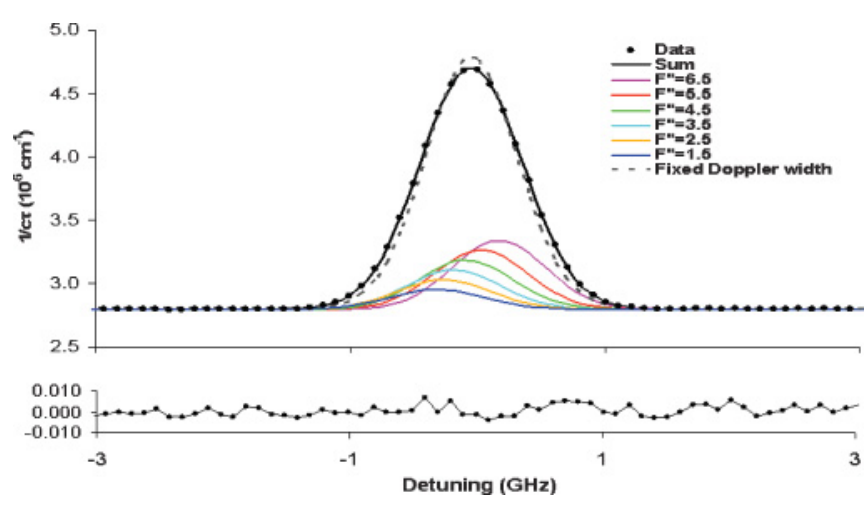

FIG. 2. (Color online) Upper panel: Measured absorption given by $(\mathrm{c} \tau)^{-1}$ where $c$ is the speed of light and $\tau$ is the measured ring-down decay time (symbols) and fitted spectrum (solid black line) for the $R 3 Q 4{ }^{16} \mathrm{O}^{17} \mathrm{O}$ transition, at $236 \mathrm{~Pa}$ (1.77 Torr) and $299.5 \mathrm{~K}$. The sum of six Voigt profiles corresponding to the hyperfine structure were fit to the measurements (solid black line) using a Levenberg-Marquardt least-squares fitting algorithm. Each of these Voigt profiles [shown in solid colored (gray) lines with larger values of $F^{\prime \prime}$ corresponding to higher intensity hyperfine components] had its relative position and intensity fixed to values calculated using the model described in the text. Each Voigt profile's Gaussian width was constrained to the theoretical Doppler width, and the pressure-broadening parameter was set equal to the measured ${ }^{16} \mathrm{O}_{2}$ value [12]. Thus, only the position of the spectral center of mass and the total integrated area of the composite spectrum were floated. Also shown is a single Voigt profile whose Doppler width was constrained to the theoretical ${ }^{16} \mathrm{O}^{17} \mathrm{O}$ value (dashed gray line). Lower panel: Fit residual for the sum of hyperfine structure. Based upon the peak height and the root-mean-square fit residual, the signal-to-noise ratio of this spectrum is $\sim 825: 1$.

both $b$ and $c$ in this fashion because these two quantities appear as differences in Eqs. (1) and (2), and as a consequence they are completely correlated for a given transition. However, $b$ and $c$ can be determined by a global multispectrum fit of spectra spanning a range of $N^{\prime \prime}$. Thus, the hyperfine model presented here was simultaneously fit to five measured spectra with $b$ and $c$ treated as global parameters. These data correspond to the $R 3 Q 4, R 4 Q 5, R 5 Q 6, R 8 Q 9$, and $R 13 Q 14$ transitions of ${ }^{16} \mathrm{O}^{17} \mathrm{O}$. Assuming the individual hyperfine transitions were Voigt profiles having the theoretical Doppler width and $J$-dependent broadening coefficients given in [12], we obtained $b=-100.93(30) \mathrm{MHz}$ and $c=129.8(40)$, which differ from the more accurate microwave measurements of Cazzoli et al. [23] by approximately $0.5 \mathrm{MHz}$ and $10 \mathrm{MHz}$, respectively. With $c$ constrained to the value determined by Cazzoli et al. (139.73 MHz), the multispectrum least-squares fit yielded $b=-101.64(10) \mathrm{MHz}$. This value of $b$ is in good agreement (difference $<200 \mathrm{kHz}$ ) with that reported by Cazzoli et al. [23]. We attribute this small difference to the finite signal-to-noise ratio of the measured absorption and to variations in the frequency of our reference laser to which the ring-down cavity was locked.

We emphasize that the hyperfine coupling constants derived from the measured splittings are directly related to important (and often difficult to calculate [30]) electronic structure properties such as the electronic density at the nucleus with nonzero nuclear spin, $|\psi(0)|^{2}$, and $\left\langle r^{-3}\right\rangle$ [31]. In the 
$b^{1} \Sigma_{g}^{+} \leftarrow X^{3} \Sigma_{g}^{-}(0,0)$ band, the hyperfine structure occurs due to the ground state; however, FS-CRDS can similarly measure hyperfine structure which is dominated by the upper state. As a result, the described technique may provide a new approach to the measurement of hyperfine coupling constants of electronic excited states, measurements which can be difficult by traditional pure rotational spectroscopy.

D. A. Long was supported by the National Science Foundation and Department of Defense. D. K. Havey acknowledges the support of the National Research Council as a postdoctoral fellow at NIST, Gaithersburg, MD. Part of the research described in this report was performed at the Jet Propulsion Laboratory, California Institute of Technology, under contract with the National Aeronautics and Space Administration (NASA). Additional support was provided by the Orbiting Carbon Observatory (OCO) project, a NASA Earth System Science Pathfinder (ESSP) mission, and the NASA Upper Atmospheric Research Program Grants NNG06GD88G and NNX09AE21G.
[1] D. J. Robichaud, J. T. Hodges, P. Masłowski, L. Y. Yeung, M. Okumura, C. E. Miller, and L. R. Brown, J. Mol. Spectrosc. 251, 27 (2008).

[2] L. S. Chen and J. Ye, Chem. Phys. Lett. 381, 777 (2003).

[3] S. Falke, E. Tiemann, C. Lisdat, H. Schnatz, and G. Grosche, Phys. Rev. A 74, 032503 (2006).

[4] G. Giusfredi, S. Bartalini, S. Borri, P. Cancio, I. Galli, D. Mazzotti, and P. De Natale, Phys. Rev. Lett. 104, 110801 (2010).

[5] D. Lisak and J. T. Hodges, Appl. Phys. B 88, 317 (2007).

[6] J. Y. Lee and J. W. Hahn, Appl. Phys. B 79, 653 (2004).

[7] S. S. Brown, H. Stark, and A. R. Ravishankara, Appl. Phys. B 75, 173 (2002).

[8] D. J. Robichaud, L. Y. Yeung, D. A. Long, M. Okumura, D. K. Havey, J. T. Hodges, C. E. Miller, and L. R. Brown, J. Phys. Chem. A 113, 13089 (2009).

[9] See Refs. [1] and [8] and the references contained therein.

[10] J. T. Hodges, H. P. Layer, W. W. Miller, and G. E. Scace, Rev. Sci. Instrum. 75, 849 (2004).

[11] J. T. Hodges and R. Ciuryło, Rev. Sci. Instrum. 76, 023112 (2005).

[12] D. A. Long, D. K. Havey, M. Okumura, C. E. Miller, and J. T. Hodges, J. Quant. Spectrosc. Radiat. Transfer (2010), doi:10.1016/j.jqsrt.2010.05.011.

[13] D. A. Long, D. K. Havey, M. Okumura, H. M. Pickett, C. E. Miller, and J. T. Hodges, Phys. Rev. A 80, 042513 (2009).
[14] D. K. Havey, D. A. Long, M. Okumura, C. E. Miller, and J. T. Hodges, Chem. Phys. Lett. 483, 49 (2009).

[15] L. Galatry, Phys. Rev. 122, 1218 (1961).

[16] K. J. Ritter and T. D. Wilkerson, J. Mol. Spectrosc. 121, 1 (1987).

[17] L. R. Brown and C. Plymate, J. Mol. Spectrosc. 199, 166 (2000).

[18] R. H. Dicke, Phys. Rev. 89, 472 (1953).

[19] D. A. Long, D. K. Havey, M. Okumura, C. E. Miller, and J. T. Hodges, J. Quant. Spectrosc. Radiat. Transfer (to be published).

[20] H. D. Babcock and L. Herzberg, Astrophys. J. 108, 167 (1948).

[21] S. L. Miller and C. H. Townes, Phys. Rev. 90, 537 (1953).

[22] P. Gerber, Helv. Phys. Acta 45, 655 (1972).

[23] G. Cazzoli, C. D. Esposti, P. G. Favero, and G. Severi, Nuovo Cimento B 62, 243 (1981)

[24] G. Cazzoli, C. D. Esposti, and B. M. Landsberg, Nuovo Cimento D 3, 341 (1984).

[25] C. H. Townes and A. K. Schawlow, Microwave Spectroscopy (Dover, New York, 1975).

[26] C. H. Townes, Phys. Rev. 71, 909 (1947).

[27] C. H. Townes and B. P. Dailey, J. Chem. Phys. 17, 782 (1949).

[28] R. A. Frosch and H. M. Foley, Phys. Rev. 88, 1337 (1952).

[29] M. Kotani, Y. Mizuno, K. Kayama, and E. Ishiguro, J. Phys. Soc. Jpn. 12, 707 (1957).

[30] B. F. Minaev, Spectrochim. Acta, Part A 60, 1027 (2004).

[31] S. L. Miller, C. H. Townes, and M. Kotani, Phys. Rev. 90, 542 (1953). 